\title{
Procédés de transformation de la mangue et niveau de connaissance des normes de qualité par les unités de production au Burkina Faso
}

\author{
Hyacinthe KANTE-TRAORE ${ }^{1 *}$, Hagrétou SAWADOGO-LINGANI ${ }^{1}$, Issiaka SEOGO ${ }^{1}$, \\ Donatien $\mathrm{KABORE}^{1}$ et Mamoudou H. DICKO ${ }^{2}$
}

\author{
I'Département Technologie Alimentaire, IRSAT/CNRST, 03 BP 7047, Ouagadougou 03, Burkina Faso. \\ ${ }^{2}$ Laboratoire de Biochimie Alimentaire Enzymologie Biotechnologie Industrielle et Bioinformatique (BAEBIB), \\ UFR/SVT Université de Ouagadougou, 03 BP7021 Ouagadougou 03, Burkina Faso. \\ *Auteur correspondant; E-mail: hyacinthe77@yahoo.fr
}

\section{RESUME}

La transformation de la mangue constitue l'activité principale de plus d'une soixantaine micro entreprises agroalimentaires au Burkina Faso. Cette activité est surtout basée sur l'utilisation de procédés traditionnels empiriques. Afin d'évaluer le niveau de connaissance des normes de qualité sur la mangue par les acteurs de la transformation et les procédés technologiques utilisés, une enquête a été réalisée auprès de 40 unités de transformation de la mangue à travers des sorties de terrains dans huit villes du pays. Les données obtenues montrent que les unités sont à $41 \%$ artisanales. La transformation porte sur les procédés de la mangue séchée, du jus/nectar, de la confiture et du sirop de mangue. Les unités (34\%) pratiquent un murissement des mangues avec le carbure de calcium et l'éthephon. Les données ont révélé que $95 \%$ des responsables des unités interviewés sont informés des normes de qualité sur la mangue; $87,5 \%$ sont engagés dans une démarche de qualité et $79 \%$ appliquent les recommandations de ces normes. Les données obtenues pourraient constituer un guide pour sensibiliser et former les acteurs sur l'importance du respect des normes de qualité; la diversification des produits finis pour fournir des produits de bonne qualité au consommateur et faire face au marché extérieur qui est fortement concurrentiel.

(C) 2017 International Formulae Group. All rights reserved.

Mots clés: Mangue, transformation, normes, procédés, qualité, Burkina Faso.

\section{Mango processing methods and level of knowledge of quality standards by actors in mango enterprise in Burkina Faso}

\begin{abstract}
Mango (Mangifera indica L.) processing is the main activity of more than sixty micro agro industrial food enterprises in Burkina Faso. This activity is mainly based on the use of traditional knowledge. To assess on the knowledge level of the quality standards of mango and its products by actors involved its technological processing a survey was conducted among 40 units of mango processing through land outlets in eight cities of Burkina Faso. Data obtained showed that the units are $41 \%$ craft. The transformation concerns the processes of dried mango, juice/nectar, and jam and mango syrup. Among units, 34\% accelerate mango ripening using calcium carbide and ethephon. The data revealed that $95 \%$ of interviewed transformers were aware of quality
\end{abstract}


standards; $87.5 \%$ were involved in quality approach; $79 \%$ implemented the recommendations of these quality standards. These findings could guide to sensitize and train stakeholders on the importance of quality standards in processing; diversification of end-products in order to provide good quality products for the consumer and also face the external market which is highly competitive.

(C) 2017 International Formulae Group. All rights reserved.

Keywords: mango, processing, process, standards, quality, Burkina Faso.

\section{INTRODUCTION}

$\mathrm{Au}$ Burkina Faso, la mangue (Mangifera indica L.) occupe une place importante et fait partie des six filières dites porteuses identifiées comme présentant un fort potentiel pour la diversification des exportations (SNV, 2014). La production nationale s'élève à environ 337101 tonnes /an pour 2274035 pieds repartis sur une superficie de 12250 hectares (CEFCOD, 2013). Elle est la plus importante production nationale fruitière et de ce fait considérée comme «premier fruit national». Parmi les quarante (40) variétés de mangue recensées dans les vergers, six sont les plus abondantes. Il s'agit des variétés Amélie, Brooks, Kent, Keitt, Lippens et Springfield (Guira et Zongo, 2006). Comme dans la plupart des pays Ouest africains, la mangue récoltée est essentiellement vendue à l'état frais à l'export et sur le marché local (ITC, 2011; Van melle et Buschmann, 2013). Une bonne partie de la récolte $(30-40 \%)$ pourrit dans les champs. Certaines mangues tombent de l'arbre avant la cueillette et d'autres sont affectées par la mouche des fruits (Vayssières et al., 2009; Ngamo et al., 2010; PAFASP, 2011; Vayssières et al., 2013). Les conditions inappropriées de cueillette et d'entreposage ainsi que l'insuffisance d'infrastructures de conservation et de techniques de traitement des mangues contribuent à augmenter les pertes post-récoltes.
Au Burkina Faso, la transformation de la mangue ne représente que $20 \%$ de la production nationale et est réalisée par une soixantaine d'unités de transformation à majorité artisanale (PAFASP, 2011). La mangue séchée est le principal produit de la transformation et représente $80 \%$ des produits transformés. Les autres produits sont les jus/nectar, la confiture, la pulpe et le sirop (Djioua, 2010 ; ITC, 2011; Kouassi, 2012). Ces produits ne sont pas assez diversifiés car la mangue est apte à diverses transformations pour obtenir des produits tels que le chutney, les pickles, les conserves de mangue au sirop, les mangues confites, la pâte de mangue, les mangues congelées ou surgelées etc. (Arnoldus et Van der pol, 2009; Arnoldus et al., 2011; MAI, 2013). Le niveau de transformation reste faible (Kouassi, 2012; Badji, 2012) à cause de la faible capacité des unités artisanales, de l'insuffisance d'infrastructures de conservation/ transformation et d'une main d'œuvre non qualifiée. Les produits finis issus de la transformation de la mangue sont confrontés à un problème de qualité du fait que certaines unités ne maîtrisent pas ou ne transforment pas suivant les normes de qualité. Cela impacte négativement l'accès aux marchés de la mangue et des produits dérivés (ITC, 2011). Bien que la transformation de la mangue soit insuffisamment exploitée, elle constitue un maillon dynamique et génère des revenus pour les acteurs et l'économie du pays. En 2007, 
600 tonnes de mangue séchée exportées ont rapporté 7 milliard de francs CFA. En 2009, environ 200 tonnes de mangue séchée exportées ont rapporté une devise de 2,5 milliard de francs CFA. Cela a porté le Burkina Faso au $1^{\text {er }}$ rang des pays exportateurs de la mangue séchée de la sous-région OuestAfricaine (Ouédraogo, 2011). De plus en plus, il existe une demande locale élevée en produits élaborés pour lesquels les consommateurs ont des exigences de qualité. Il existe également une demande de produits finis de qualité pour les marchés à l'exportation, dont l'accès est totalement conditionné par le respect de normes qui sont de plus en plus strictes. Les unités de transformation devraient travailler davantage à diversifier et à améliorer la qualité de leurs produits en se conformant aux exigences de la réglementation régionale et internationale. Le présent travail s'inscrit dans cette lancée en évaluant le niveau de connaissance des normes de qualité par les transformateurs, afin d'améliorer les procédés de production et diversifier les produits dérivés.

\section{MATERIEL ET METHODES}

L'approche méthodologique adoptée dans la présente étude a consisté à l'identification des zones d'étude, à l'élaboration de l'outil de collecte des données, à la collecte des données sous forme d'enquête/entretien, à l'analyse et à l'exploitation de ces données collectées.

\section{Zone d'étude}

L'étude a été réalisée au Burkina Faso dans les principales zones de production de la mangue (Figure 1) et d'implantation d'unités et d'industries de transformation de la mangue. Elle a concerné cinq régions et huit villes. Il s'agit principalement des villes de Banfora et Nyangologo dans la région des Cascades, Bobo-Dioulasso, Orodara et Toussiana dans la région des Hauts bassins, Ouagadougou dans la région du centre, Réo dans la région du Centre-Ouest et Ouahigouya dans la région du Nord.

\section{Outil de collecte}

L'outil de collecte des données est un questionnaire (fiche d'enquête) conçu par une équipe composée d'un ingénieur de recherche, un technicien supérieur de recherche, un sociologue et un statisticien. Ce questionnaire a été soumis à un comité pour vérifier son adaptation et le valider. Une recherche bibliographique a été ensuite menée pour recenser toutes les unités (Tableau 1) de transformation de la mangue connues au Burkina Faso. Une sortie de terrain a été effectuée auprès des unités qui ont accepté l'enquête dans les localités suscitées. L'enquête a permis de recueillir des informations sur les quatre points suivants: les caractéristiques des unités de transformation, les variétés de mangue utilisées, les techniques de stockage et de murissement pratiquées, les produits transformés et leurs procédés de production, et le niveau de connaissance des normes/spécifications de qualité par les acteurs du maillon de la transformation. La collecte de ces informations a été effectuée par quatre équipes d'enquêteurs réparties dans chaque zone d'étude.

\section{Traitement des données}

L'élaboration du masque de saisie et l'introduction des données a été faite avec le logiciel CSPRO 5. 


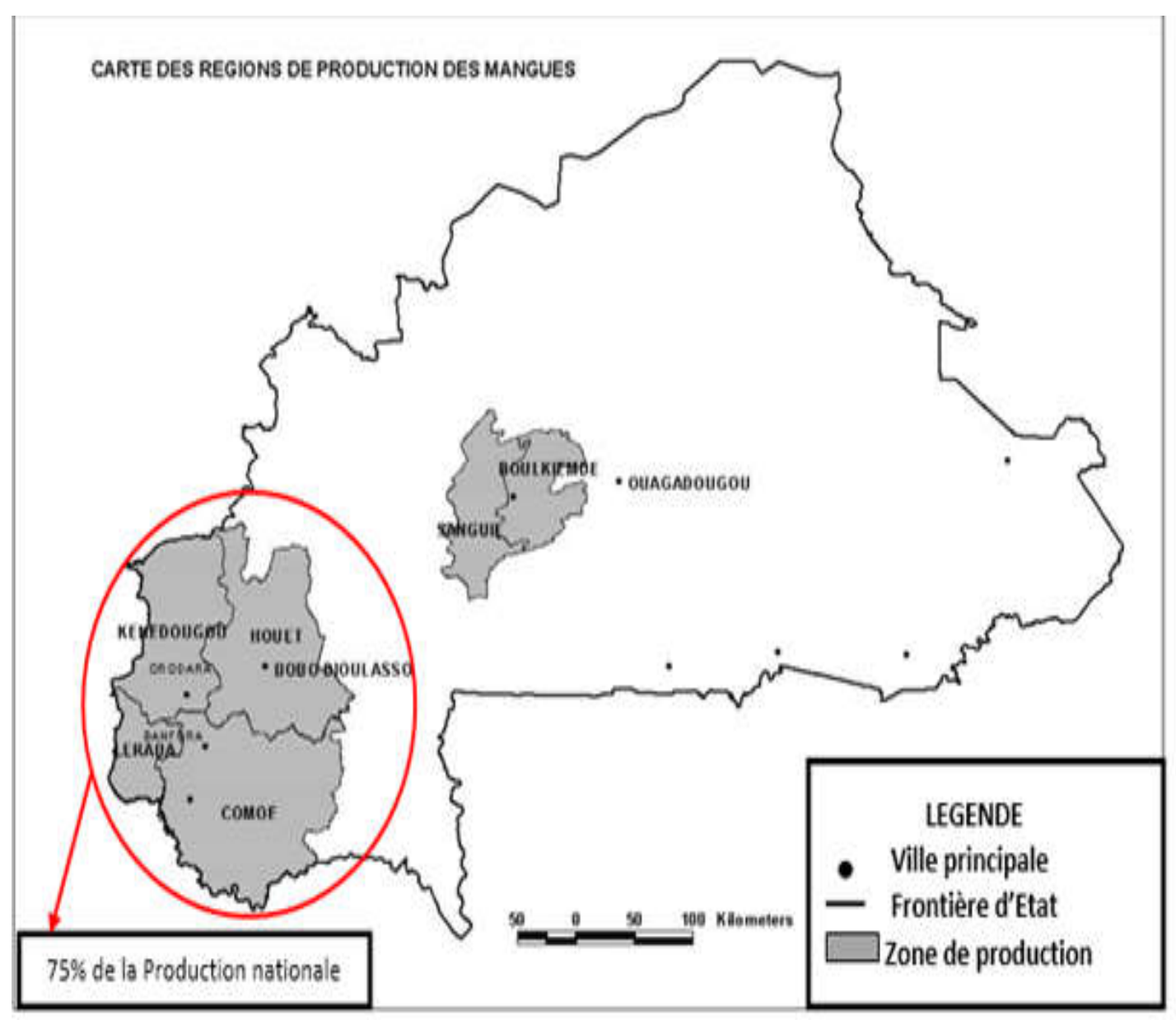

Figure 1: Principales zones de production de la mangue au Burkina Faso.

Tableau 1: Nombre d'unités de transformation de la mangue recensées.

\begin{tabular}{ll}
\hline Localité & Nombre d'unités \\
\hline Cascades & 8 \\
Centre-Ouest & 1 \\
Hauts-Bassins & 45 \\
Centre & 10 \\
Nord & 1 \\
Total & $\mathbf{6 5}$ \\
\hline
\end{tabular}




\section{RESULTATS}

L'enquête a été réalisée dans 40 unités de transformation de la mangue. Les personnes interviewées étaient à $70,00 \%$ des hommes contre $30,00 \%$ de femmes. Ces personnes sont pour la plupart les promoteurs $(70,00 \%)$ des unités, des responsables de production $(15,00 \%)$ et rarement des employés $(2,50 \%)$. L'enquête a révélé que plus de la moitié des personnes interviewées $(57,50 \%)$ ont plus de 45 ans, $27,50 \%$ ont un âge compris entre 36 et 45 ans et $15,00 \%$ se situent dans la tranche d'âge de 26 à 35 ans. S'agissant de l'éducation, $47,50 \%$ des répondants ont un niveau d'instruction universitaire et $30,00 \%$ le niveau de l'enseignement secondaire. Les autres personnes interviewées représentent respectivement $10,00 \%$ et $12,50 \%$ des niveaux primaires et post-primaires.

\section{Typologie et caractéristiques des unités de transformation enquêtées}

Parmi les 40 unités de transformation enquêtées (Figure 2), 60,00\% sont localisées dans la région des Hauts bassins, $22,50 \%$ dans la région du Centre, $12,50 \%$ proviennent de la région des Cascades et $2,50 \%$ proviennent respectivement du Centre-Ouest et du Nord. Les données de l'enquête ont révélé que ces unités sont composées d'unités artisanales $(41,00 \%)$; d'unités semi industrielles $(56,00 \%)$ et d'unités industrielles représentant $3,00 \%$. L'ancienneté de ces unités de transformation de la mangue varie de 3 ans à 32 ans.

Les données ont également montré que $46,20 \%$ de ces unités sont des propriétés individuelles, $28,20 \%$ représentent des groupements/associations, $\quad 5,10 \% \quad$ sont respectivement des sociétés anonymes et des petites et moyennes industries/ou entreprises (PMI/PME), 10,30\% de ces unités sont des coopératives et $5,10 \%$ des sociétés à responsabilité limité (SARL).

Plus de la majorité des unités $(95,00 \%$ correspondant à 38 unités) effectuent une production exclusivement destinée à la consommation humaine et $5,00 \%$ des unités effectuent une production destinée à la fois à la consommation humaine et à l'alimentation des animaux. La commercialisation des produits transformés est assurée par les unités elles-mêmes. La capacité d'employabilité est très variable car le nombre de personnes employées dans ces unités varient de 5 à 800 avec une moyenne de 97 personnes.

\section{Variétés de mangue utilisées dans les unités de transformation enquêtées}

Les variétés de mangue les plus utilisées dans les unités de transformation sont représentées au niveau des Figures 3 et 4 . Les données de l'enquête montrent qu'on trouve cinq variétés les plus utilisées dans la transformation. Ce sont: Amélie, Brooks, Kent, Lippens et Springfield. La variété Amélie est majoritairement la plus transformée dans toutes les unités enquêtées. Elle est suivie par ordre décroissant des variétés Brooks, Lippens, Kent et Springfield. La dernière variété est rarement rencontrée dans la transformation au niveau des unités. Plusieurs raisons expliquent le choix des variétés. On peut citer comme exemple la demande du client qui est de 51,00\% pour la variété Amélie et $17,00 \%$ pour la variété Brooks; la disponibilité de la variété qui est de $32,00 \%$ pour l'Amélie et $11,00 \%$ pour la Brooks; la facilité de transformation qui est de $29,00 \%$ pour l'Amélie et $7,00 \%$ pour la Brooks.

\section{Pratiques d'entreposage et de mûrissement de la mangue dans les unités enquêtées}

Les techniques de mûrissement appliquées sur les mangues qui arrivent dans les unités de transformation sont de deux sortes à savoir le mûrissement naturel et le mûrissement utilisant des produits chimiques. Les données ont révélé que $15,00 \%$ des unités interviewées ne disposent pas d'infrastructures de stockage/entreposage des mangues qu'elles utilisent pour la transformation. Fort heureusement $85,00 \%$ en disposent et pratiquent le mûrissement des mangues avant la transformation. La capacité des infrastructures de stockage/entreposage disponibles varie de 1000 à $800000 \mathrm{Kg}$ avec 
une durée de stockage allant de 2 à 10 jours. La durée moyenne de stockage est de 6,60 jours. Parmi les unités de transformation effectuant le mûrissement des mangues, $34,00 \%$ pratiquent le mûrissement accéléré à l'aide de produits chimiques qui sont soit le carbure de calcium, soit l'éthephon.

Le processus de l'utilisation du carbure de calcium se fait en disposant les mangues triées dans des caisses superposées les unes sur les autres. Le carbure de calcium mis dans une boîte perforée, aspergé d'eau puis placée en dessous des caisses. L'ensemble du dispositif est recouvert avec une bâche pour le mûrissement. L'utilisation de l'éthephon diffère légèrement de celle $\mathrm{du}$ carbure de calcium du fait qu'il soit liquide. Une certaine quantité est diluée dans de l'eau puis aspergée directement sur les mangues qui sont ensuite recouvertes par une bâche. Les autres (66\%) pratiquent le mûrissement naturel des mangues qui consiste à entreposer les mangues triées dans une salle ou dans des bacs, les recouvrir avec une bâche puis laisser mûrir. Ce processus est plus lent (allant jusqu'à 3 semaines parfois selon les variétés).

\section{Procédés de transformation de la mangue dans les unités enquêtées}

Les données obtenues ont révélé la pratique de différents procédés de transformation dans les unités. Les technologies les plus rencontrées sont celles de la mangue séchée, suivi de celle de la production de jus/nectar de mangue, de confiture/marmelade, de sirop de mangue et de pulpe/purée de mangue. Le diagramme des produits les plus transformés est représenté au niveau de la Figure 5. La mangue séchée est le premier produit dérivé de la mangue au Burkina Faso. Les produits séchés sont suivis respectivement du nectar, de la pulpe et du sirop de mangue. En plus de ces technologies, il y a également des technologies moins connues comme la fabrication de "moût" de mangue, de "gâteau" de mangue, de "galette" et de "rouleau" de mangue. Les équipements utilisés dans la transformation sont constitués d'équipements artisanaux (bassines, marmites, couteaux, cuvettes etc.) et d'équipements industriels (table de découpe, éplucheuses en inox, cuvettes, couteaux et plateaux en inox, centrifugeuse, pasteurisateur, réfractomètre, dateuse, remplisseuse...).

La durée de conservation des produits issus des quatre procédés décrits, varie entre 6 et 18 mois. La durée de conservation est déterminée par un suivi de conservation avec des analyses microbiologiques et physicochimiques du produit selon les dires des enquêtés par usage de données bibliographiques, ou déterminée arbitrairement. Dans ce cas, après production, la transformatrice donne une durée de vie sans suivi de la conservation ni de recherche bibliographique. En effet, parmi les 40 unités enquêtées, $47,80 \%$ effectuent un suivi de conservation (le plus souvent une observation des produits à l'œil nu pendant 6 mois) à la suite duquel elles fixent la durée de vie du produit; $21,70 \%$ se basent sur les données bibliographiques et $30,40 \%$ fixent la durée de vie de leur produit arbitrairement.

\section{Niveau d'information et de connaissance des normes de qualité par les acteurs de la transformation de la mangue}

Les acteurs intervenant dans la transformation de la mangue devraient pratiquer cette activité suivant certaines normes internationales de qualité. Ainsi, selon les données collectées, $95,00 \%$ des unités interviewées sont au courant des normes régulant la transformation de la mangue. Cependant, d'une unité à une autre, les connaissances et les applications de ces normes diffèrent. Les Normes Burkinabè (ABNORM) sur la mangue fraîche, la mangue séchée et le jus/nectar de mangue, l'exigence pour la mangue séchée biologique, les bonnes pratiques d'hygiène et de fabrication (BPH/BPF), le codex alimentaire sur la mangue, la norme suisse sur les fruits et légume séchés et HACCP sont les normes les plus connues des acteurs. Parmi les unités enquêtées, $87,50 \%$ sont inscrites dans une démarche qualité qui peut être soit la certification pour la transformation de la 
mangue biologique, la certification selon le système HACCP, la certification selon la norme ISO 22000 ou encore les bonnes pratiques d'hygiène/bonnes pratiques de fabrication (BPH/BPF) et le commerce équitable. Parmi les unités qui connaissent ces normes, $79,00 \%$ font des efforts pour les appliquer et pensent être dans les règles. Cependant elles stipulent rencontrer des difficultés au niveau de leur application. Les autres unités $(21,00 \%)$ connaissent l'existence des normes et leurs exigences en matière d'assurance qualité mais ne les appliquent pas. Les raisons évoquées pour justifier cette non application sont entre autres l'absence, l'indisponibilité ou la cherté des documents des normes; l'absence de formation sur l'utilisation des normes; l'analphabétisme d'une grande partie du personnel employé dans les unités de transformation; l'utilisation d'équipements non adaptés à la transformation; le coût élevé des analyses microbiologiques des produits transformés et les difficultés liés au remplissage des cahiers de charge.

\section{Contrôle de la qualité des produits dérivés de la mangue dans les unités}

Le contrôle de la qualité des produits transformés est effectué dans les laboratoires d'analyse disponibles au Burkina Faso. Les unités de transformation ont souvent recours à ces laboratoires pour l'analyse des paramètres physico-chimiques, microbiologiques, nutritionnels et sensoriels de leurs produits. Ainsi $80 \%$ des acteurs affirment avoir déjà fait analyser leurs produits dans un laboratoire pour au moins un paramètre de qualité. Parmi eux, $72 \%$ reconnaissent avoir effectué des analyses physico-chimiques et microbiologiques, $25 \%$ ont effectué uniquement des analyses physico-chimiques et $3 \%$ ont effectué uniquement des analyses microbiologiques sur les produits. Toutes les unités interviewées reconnaissent que ces analyses ne sont pas faites de façon permanente.

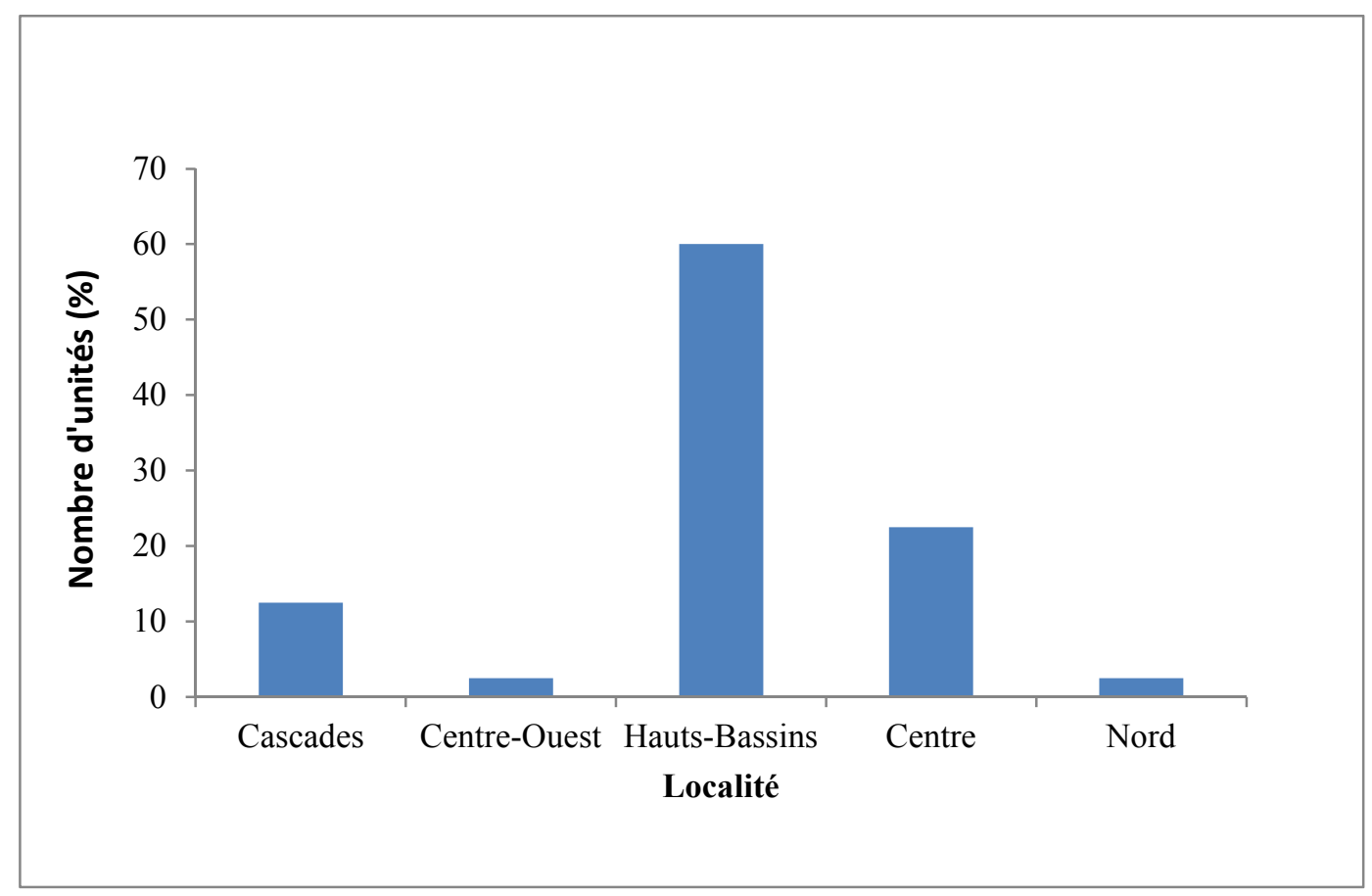

Figure 2: Répartition des unités de transformation selon les localités. 


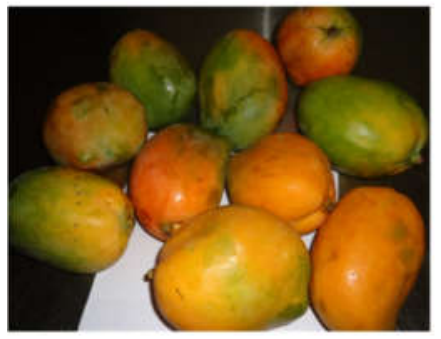

Variété Amélie

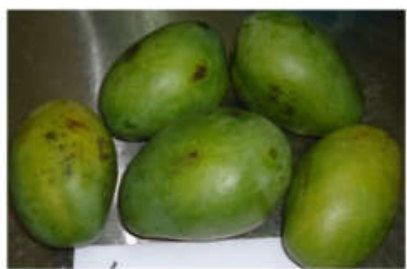

Variété Brooks

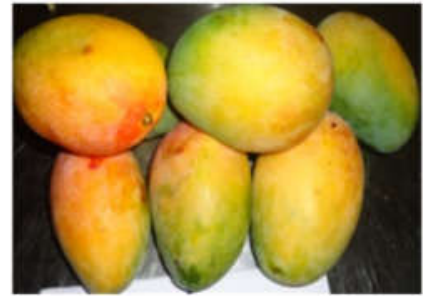

Variété Lippens

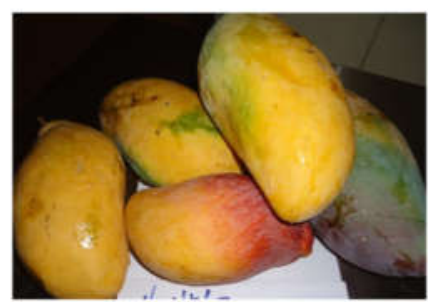

Variété Springfield

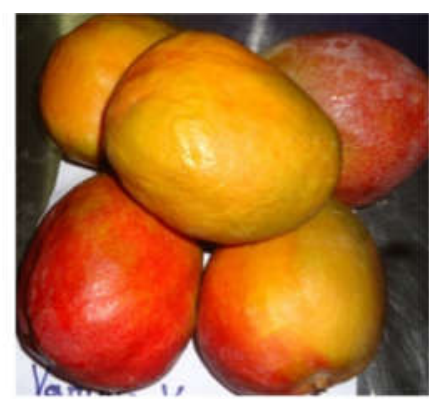

Variété Kent

Figure 3 : Principales variétés utilisées dans les unités de transformation au Burkina Faso.

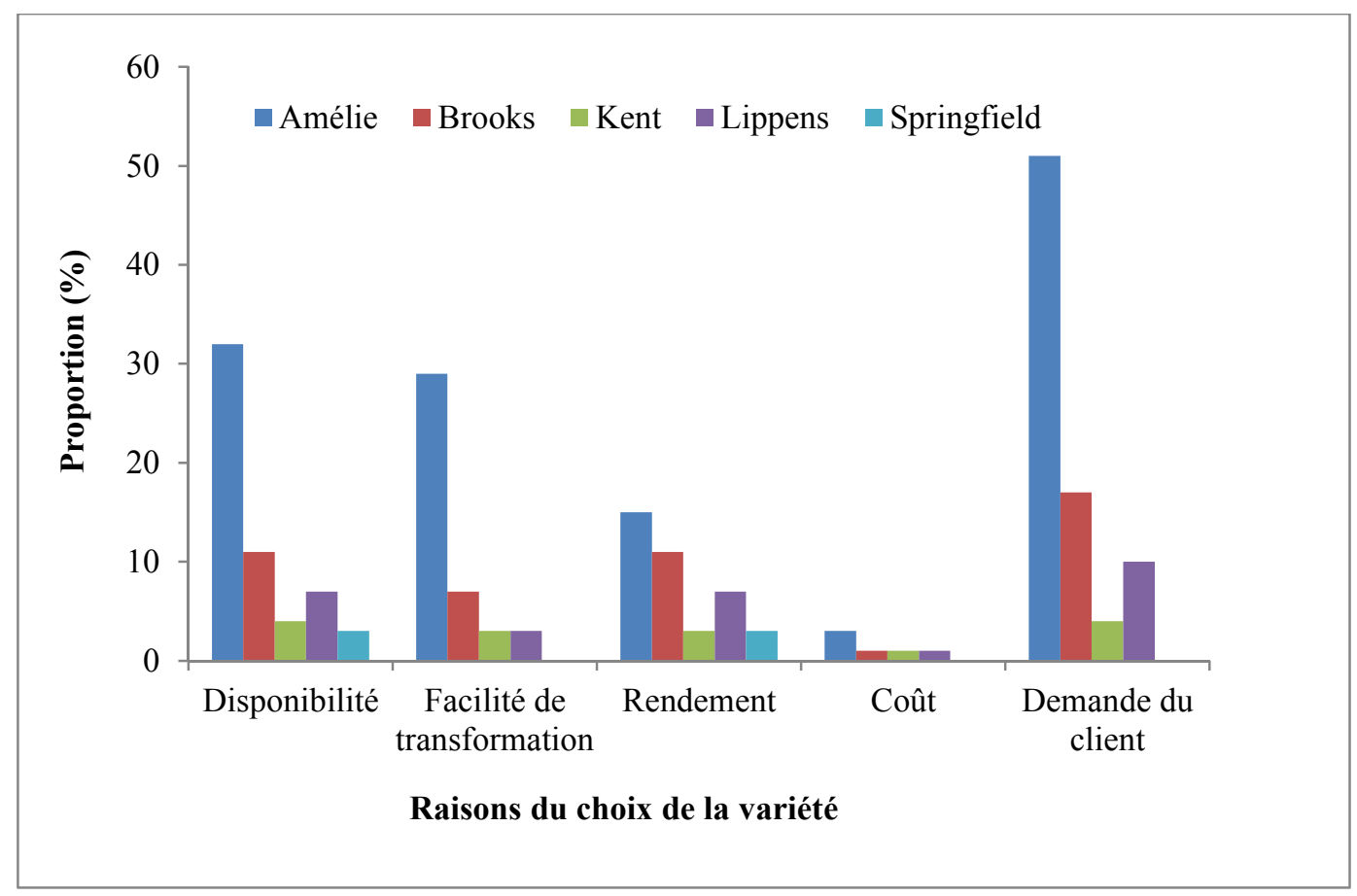

Figure 4: Raisons du choix de ces variétés utilisées dans les unités de transformation au Burkina Faso. 

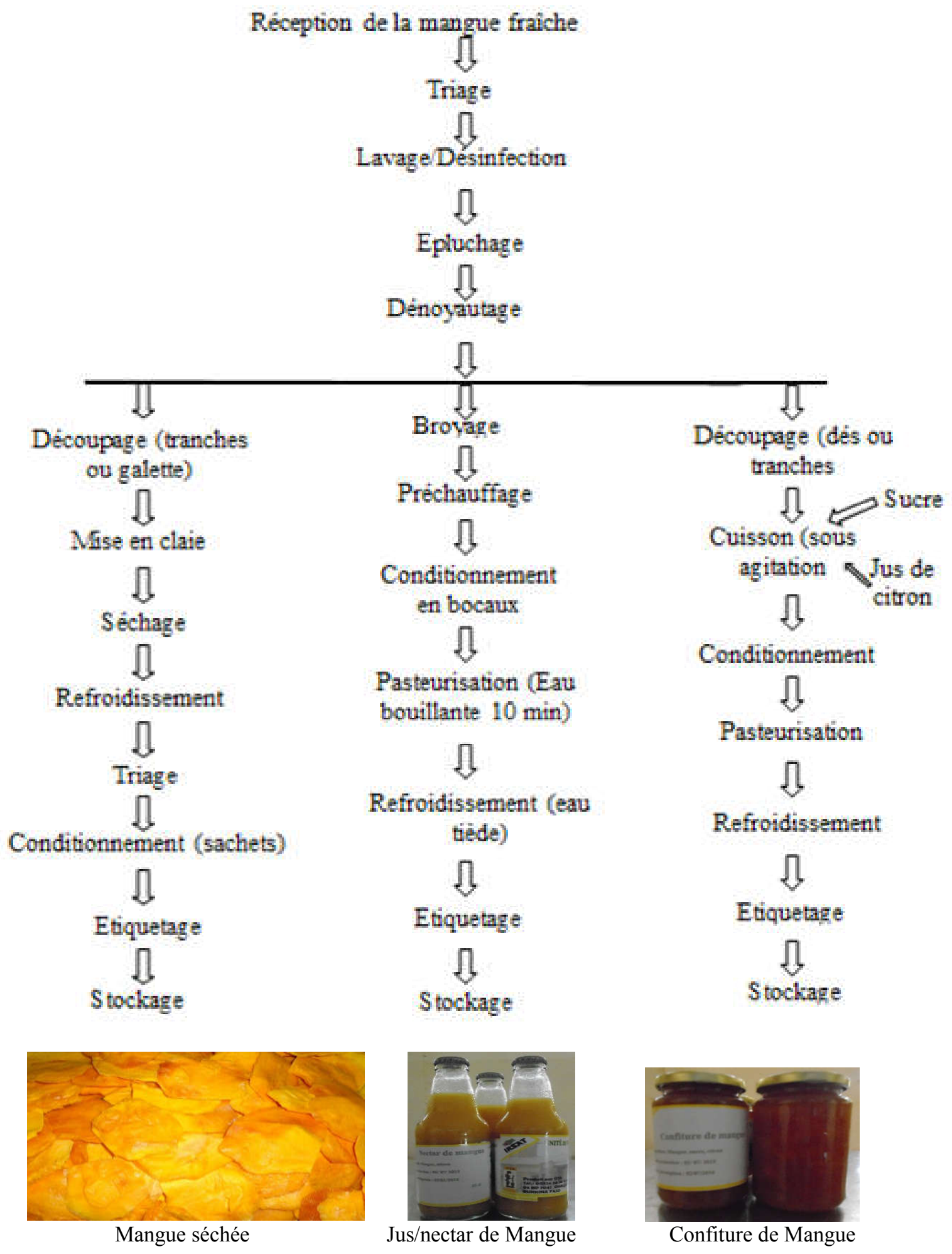

Figure 5: Diagramme de fabrication des trois produits fars. 


\section{DISCUSSION}

Les données obtenues au cours de cette étude ont montré qu'il existe trois types d'unités de transformation de la mangue au Burkina Faso: les unités artisanales, les unités semi-industrielles et les unités industrielles. Cette typologie est similaire à celle recensée au Mali à travers l'étude diagnostique du secteur de la transformation des produits agricoles (PCDA, 2005). On constate une faible implication des jeunes (15\%) parmi le personnel des unités travaillant dans l'activité de transformation de la mangue.

Les variétés de mangue utilisées dans la transformation sont majoritairement celles que l'on rencontre le plus dans les vergers des pays Ouest-africains (Rey et al., 2004; Guira et Zongo, 2006). La variété Amélie est la plus utilisée au Burkina Faso à cause de sa précocité car elle est disponible dès le mois d'avril. Elle est aussi fortement appréciée par les consommateurs. Sa chair, d'une couleur orangée, ne contient pas de fibre et est très édulcorante d'où sa plus grande utilisation dans les unités de transformation. La variété Kent est une variété colorée de couleur jaune verdâtre parfois tachetée de rouge foncé ou rose violet. Elle a été introduite au Burkina Faso pour répondre aux besoins de l'exportation. Sa chair de couleur jaune intense est fondante, juteuse et sans fibre. Cette variété colorée est beaucoup appréciée et la plus recherchée sur le marché des pays de l'Union Européenne où elle est considérée comme la référence pour les mangues vendues (CBI, 2015). Elle est disponible mais pas facilement accessible car elle est vendue chère sur le marché local d'où sa faible utilisation dans la transformation de la mangue. Seule l'usine DAFANI utilise la variété Kent dans la transformation du fait de son implantation à Orodara (cœur du verger burkinabè). Elle peut traiter directement avec les producteurs et a un pouvoir d'achat élevé. Cependant la variété Kent est utilisée au Mali pour le séchage, la fabrication des jus/nectars et confiture (PCDA, 2005). La faible utilisation de la variété Springfield peut être due à sa qualité car cette variété est fibreuse.
La pratique du murissement des mangues par utilisation de produits chimiques que sont l'éthylène $\left(\mathrm{C}_{2} \mathrm{H}_{4}\right)$, l'acétylène $\left(\mathrm{C}_{2} \mathrm{H}_{2}\right)$, l'éthrel/éthéphon $\left(\mathrm{C}_{2} \mathrm{H}_{6} \mathrm{ClO}_{3} \mathrm{P}\right)$ et le carbure de calcium $\left(\mathrm{CaC}_{2}\right)$ est admise à condition de maîtriser le dosage et les conditions d'application (De la Cruz et Garcia, 2002; MAI, 2013). Le dosage est fait en fonction de la variété, de la durée de traitement et de la température. En Inde, l'utilisation de l'éthrel et du carbure de calcium ( $4 \mathrm{~g} / \mathrm{kg}$ de fruit) pour mûrir la variété Dashehari, donne à cette variété un goût agréable, une couleur attractive, augmente la teneur en caroténoïdes, en extrait sec soluble et en sucres édulcorants. Toutefois une baisse de la teneur en acide ascorbique (vitamine C) est observée (MAI, 2013). L'utilisation de $6 \mathrm{~g}$ de carbure de calcium par kilogramme de fruits suivi d'un traitement à l'éthrel à $800 \mathrm{ppm}$ améliore la saveur de la variété Langra (MAI, 2013). L'utilisation de l'acétylène à une concentration de $0,4 \mathrm{ml} / 1$ à $25{ }^{\circ} \mathrm{C}$ pendant 24 heures ramollie la mangue et lui donne une belle couleur uniforme mais la saveur reste pauvre (MAI, 2013). C'est l'acétylène libéré qui est responsable du murissement accéléré des mangues. On estime qu'un kilogramme de carbure de calcium libère 350 litres d'acétylène (Darne, 2001). L'acétylène est un gaz très volatil et très inflammable. A forte concentration, il provoque une asphyxie. L'homme devient inconscient s'il est exposé à une concentration de $35 \%$ d'acétylène pendant 5 min. Les effets d'intoxication à l'acétylène se manifestent par des céphalées, des vertiges, des nausées, une incoordination motrice et parfois une perte de conscience (Picot et Montandon, 2013). L'éthéphon ou l'éthrel $\left(\mathrm{C}_{2} \mathrm{H}_{6} \mathrm{ClO}_{3} \mathrm{P}\right)$ se décompose en éthylène, phosphate et ion chlorure dans des solutions aqueuses à $\mathrm{pH}>4$ (Picot et Montandon, 2013). L'éthéphon est économiquement important car il se décompose en éthylène qui est un régulateur de la croissance des plantes ou un accélérateur du murissement des fruits climactériques tels que la mangue. $\mathrm{Au}$ Burkina Faso, le carbure de calcium ainsi que l'éthrel sont les produits chimiques les plus 
utilisés pour le murissement des mangues par les vendeuses de la mangue fraîche et par certains transformateurs. Les doses et les conditions d'utilisation de ces produits ne sont pas maîtrisées par ces utilisateurs. Etant donné que ces produits ont des conséquences néfastes sur la santé humain, il est donc urgent qu'une sensibilisation soit menée auprès des acteurs sur les méthodes et les conditions d'utilisation de ces produits chimiques afin qu'ils soient bien maitrisés.

Le manque d'infrastructures de stockage des mangues dans les unités révèle leur faible capacité de transformation. En effet, au Burkina Faso comme dans les pays de la sous-région, les industries de transformation agroalimentaire, particulièrement celles de la transformation des fruits ne sont pas très développées. Lorsqu'elles existent, elles pratiquent des procédés artisanaux de transformation. Ces procédés artisanaux ont un impact sur les produits transformés. En effet, on observe une irrégularité de la qualité d'une production à une autre faute de maitrise des bonnes pratiques ou du respect strict des normes. Des progrès d'amélioration du maillon de la transformation sont en train d'être réalisés au Burkina Faso ainsi que dans les pays de la sous-région. Par exemple au Burkina Faso, depuis 2007, il existe une usine de transformation de la mangue dénommée «DAFANI» (PAFASP, 2011). De même au Sénégal, il existe une usine de production de vinaigre à base de mangue (Tounkara et al., 2011). Au Mali, depuis 2009, une mise en place d'une usine de production de la purée de mangue a été réalisée par Abt Associates Investment Analysis (Abt Associates Investment Analysis, 2009).

Les procédés de fabrication rencontrés dans les unités sont ceux relatés par Kameni et al. (2003), Vayssiere et al. (2008), MAI (2013). Ces procédés sont assez bien maîtrisés par les unités de transformation surtout celui de la mangue séchée au Burkina Faso. Cependant les produits dérivés de la mangue ne sont pas assez diversifiés alors qu'il existe plusieurs autres technologies de transformation de la mangue dans les pays comme l'Inde (MIA, 2013). Les promoteurs des unités de transformation pourraient s'inspirer de ces technologies pour diversifier les produits dérivés de la mangue et lui apporter une valeur ajoutée. Le contrôle qualité des produits transformés de la mangue revêt être une problématique pour les unités.

$\mathrm{Au}$ regard des données recueillies sur la durée de vie des produits, $47,8 \%$ des transformateurs/transformatrices effectuent un suivi de conservation pour déterminer cette durée de vie. Ce suivi de conservation correspond le plus souvent à une observation à l'œil nu des produits transformés sur une période de six ou 12 mois. L'observation à l'œil nue est quelque fois appuyée par des analyses microbiologiques. Cela est inquiétant car ces produits dont la qualité sanitaire n'est pas confirmée par des analyses physicochimiques et microbiologiques, pourraient être impropres à la consommation. Cette inquiétude montre qu'il y a un énorme travail de sensibilisation à faire sur l'application des normes de qualité dans la transformation des produits agroalimentaires ainsi que sur l'importance des analyses physico-chimiques et microbiologiques de ces produits. Il est également important que les structures d'appui technique comme les laboratoires collaborent avec les unités de transformation pour la détermination de la durée de vie des produits afin de garantir la qualité des produits mis sur le marché. C'est pourquoi les efforts fournis par certaines unités inscrites dans des démarches de certification et l'application des bonnes pratiques d'hygiènes et de fabrication sont à encourager.

\section{Conclusion}

Cette étude a permis de faire l'état des lieux des unités de transformation de la mangue au Burkina Faso et d'évaluer le niveau de connaissance des normes/spécifications de qualité par les promoteurs. Les principaux procédés technologiques, les variétés de mangue et les pratiques de murissement utilisé dans les unités de transformation sont maintenant 
identifiées. Bien que plus de la majorité des transformateurs/transformatrices soit informée sur les normes de qualité régissant la transformation de la mangue et les applique, la qualité des produit finis reste à parfaire. Ainsi, il y a lieu de continuer la sensibilisation et la formation des acteurs pour une amélioration des procédés technologiques et la maîtrise de la qualité des produits. Il importe aussi de créer des centres d'incubation pour suivre ces transformateurs dans leurs activités de transformation afin de permettre à la filière mangue d'être plus compétitive. Les données collectées pourront servir aux décideurs et porteurs de projets d'innovation au niveau de la transformation pour une meilleure orientation de leur intervention.

\section{CONFLIT D'INTERETS}

Les auteurs déclarent qu'il $\mathrm{n}$ y a aucun conflit d'intérêts.

\section{CONTRIBUTIONS DES AUTEURS}

HKT a élaboré l'outil de collecte des données, collecté les données et a rédigé le manuscrit. HSL a corrigé l'outil de collecte des données, supervisé la collecte des données et a corrigé le manuscrit. IS a participé à l'élaboration de l'outil de collecte des données, collecté et traité les données. KD a participé à la correction du manuscrit. MHD a participé à la correction du manuscrit.

\section{REMERCIEMENTS}

Cette étude a été financée par la Banque Mondiale à travers le Programme de Productivité Agricole en Afrique de l'Ouest (PPAAO/WAAPP) et son Centre National de Spécialisation en Fruits et Légumes (CNSFL). Les auteurs remercient le personnel du Département Technologie Alimentaire (DTA) ayant contribué à la collecte des données sur le terrain, ainsi que les unités de transformation de la mangue.

\section{REFERENCES}

Arnoldus M, Van der Pol F, Ravry C. 2011. Affaire juteuse ou déception amère: quel est l'avenir des produits dérivés de la mangue au Burkina Faso et au Mali? Perspectives et stratégies pour la production et commercialisation de la mangue séchée, de la pulpe et du jus de mangue. Institut Royal des Tropiques (KIT), Amsterdam, Pays-Bas ISBN: 978 $9060221422,143 \mathrm{p}$

Arnoldus M, Van der Pol F. 2009. Amélioration des performances de la filière des produits transformés de la mangue au Burkina Faso et au Mali. Une étude pour la Banque Mondiale, le PCDA et le PAFASP, financée par l'Union Européenne, $116 \mathrm{p}$.

Abt associates investment analysis. 2009. Mango Pulp and Nectar Processing in Mali: A Technical and Financial Analysis for the Malian Investor, USAID, $42 \mathrm{p}$.

Badji O. 2012. Revue nationale sur la valorisation non Alimentaire des mangues au Sénégal. COLEACP-PAEPARD, décembre 2012, $23 \mathrm{p}$.

CBI (Centre for the Promotion of imports from developing countries). 2015. Caractéristiques produits pour les mangues fraîches. www.cbi.eu/disclaimer. Consulté le 13/08/2015.

CEFCOD (Centre d'Etude, de Formation et de Conseil en Développement). 2013. Situation de référence des principales filières agricoles au Burkina Faso. Rapport version finale, Ministère de l'Agriculture et de la Sécurité Alimentaire, $74 \mathrm{p}$.

Darne F. 2001. Du carbure de calcium à l'acétylène. Groupe d'études techniques de $l^{\prime} E F S$. Spelunca $\mathrm{n}^{\circ} 83-3^{\text {ème }}$ trimestre, $8 \mathrm{p}$.

De la Cruz JM, Garcia HS. 2002. Mango: Post-harvest Operations. AGSI/FAO: Danilo Mejia, PhD (Technical), Beverly Lewis (Language \& Style). Last reviewed, $70 \mathrm{p}$.

Djioua T. 2010. Amélioration de la conservation des mangues 4ème gamme par application de traitements thermiques et utilisation d'une conservation sous atmosphère modifiée. Thèse de doctorat, Académie d'Aix-Marseille Université d'Avignon et des Pays de Vaucluse, $169 \mathrm{p}$.

Guira M, Zongo JD. 2006. Etude de la distribution des variétés cultivées dans les vergers de manguier de l'Ouest du Burkina Faso. Sciences et Techniques, série Sciences Naturelles et Agronomie, 28(1 et 2): 63-72.

ITC (Centre du Commerce International). 2011. Mangue, Service des Nouvelles des 
Marchés (MNS). Bulletin MNS, ECOWAS TEN, $26 \mathrm{p}$.

Kameni A, Mbofung CM, Ngnamtam Z, Doassem J, Hamadou L. 2003. Aptitude au séchage de quelques variétés de mangue cultivées au Cameroum: Amélie, Zill, Irwin, Horé Wandou. In Savanes africaine: des espaces de mutation, des acteurs face à de nouveaux défis, Jamin JY, Seini BL, Floret C (Eds). Actes du colloque, mai 2002, Garoua, Cameroun. Prasac, N'Djamena, Tchad-Cirad, Montpellier, France, $9 \mathrm{p}$.

Kouassi OA. 2012. Revue nationale pour identifier les initiatives de valorisation non alimentaire de la mangue en Côte d'ivoire. $\mathrm{N}^{\circ}$ 12/COLEACP PAEPARD-01/BNA-12. Septembre-décembre 2012, $43 \mathrm{p}$.

MAI (Ministry of Agriculture of India). 2013. Post-harvest profile of mango. Department of agriculture \& cooperation. Directorate of marketing \& inspection Branch head office Nagpur, $141 \mathrm{p}$.

Ngamo Tinkeu L, Ladang D, Vayssieres JF, Lyannaz JP. 2010. Diversité des espèces de mouches des fruits (Diptera : Tephritidae) dans un verger mixte dans la localité de Malang (Ngaoundéré, Cameroun). Int. J. Biol. Chem. Sci., 4(5): 1425-1434. Available online at http://ajol.info/index.php/ijbcs

Ouédraogo SN. 2011. Dynamique spatio temporelle des mouches des fruits (diptera, tephritidae) en fonction des facteurs biotiques et abiotiques dans les vergers de manguiers de l'ouest du Burkina Faso. Thèse de doctorat spécialité écophysiologie. Université Paris Est, Ecole doctorale science de la vie et de la santé, $184 \mathrm{p}$.

PAFASP (Programme d'Appui aux Filières Agro-Sylvo-Pastorales). 2011. Analyse des chaines de valeur ajoutée des filières Agro-Sylvo-Pastorales: bétail/viande, volaille, oignon et mangue. CAPES, rapport définitif, $212 \mathrm{p}$.

Picot A, Montandon F. 2013. Ecotoxicochimie Appliquée aux Hydrocarbures. TEC \& DOC. Lavoisier: Paris ; 281-287.

PCDA (Programme Compétitivité et Diversification Agricoles). 2005. Etude diagnostique du secteur de la transformation des produits agricoles. Rapport final, LTA/IER, janvier 2005, Mali, $106 \mathrm{p}$.

Tounkara LS, Beye C, Cisse N, Laurent L, Diop A. 2011. Réduction des pertes postrécoltes de la mangue par la production de vinaigre. ITA-CWBI-FNRAA, $8 \mathrm{p}$.

Rey JY, Diallo TM, Vannière H, Didier C, Kéita S, Sangaré M. 2004. La mangue en Afrique de l'Ouest francophone: variétés et composition variétale des vergers. Fruits, 59. DOI: 10.1051/fruits:2004018: 191-208

SNV (Organisation Néerlandaise de Développement). 2014. Projet d'appui à la commercialisation de mangues séchées et de noix de cajou transformés. Mémorandum d'accord entre le Bureau des Nations Unies pour les services d'appui aux projets et le Gouvernement du Burkina Faso pour l'exécution du Projet de Catégorie 2 du Cadre Intégré Renforcé, $123 \mathrm{p}$.

Van Melle C, Buschmann S. 2013. Analyse comparative des chaînes de valeur de la mangue au Bénin, au Ghana et au Burkina Faso, dans : Reconstruire le potentiel alimentaire de l'Afrique de l'Ouest, Elbehri A (ed). FAO/FIDA, 34 p.

Vayssieres JF, Korie S, Coulibaly T, Temple L, Boueyi S. 2008. The mango tree in northern Benin (1): cultivar inventory, yield assessment, early infested stages of mangos and economic loss due to the fruit fly (Diptera Tephritidae). Fruits, 63: 1-22.

Vayssières J-F, Korie A, Ayegnon D. 2009. Correlation of fruit fly (Diptera: Tephritidae) infestation of major mango cultivars in Borgou (Benin) with abiotic and biotic factors and assessment of damage. Crop Prot., 28: 477-488.

Vayssieres JF, Sinzogan A, Adandonon A, Van Mele Paul, Korie S. 2013. Ovipositional behaviour of two mango fruit fly species (Diptera Tephritidae) in relation to Oecophylla cues (Hymenoptera Formicidae) as compared to natural conditions without ant cues. Int. J. Biol. Chem. Sci., 7(2): 447-456. Available online at http://ajol.info/index.php/ijbcs. 